\title{
A Review of the Vascular Anatomy of the Optic Nerve Head and Its Clinical Implications
}

Andrew S. McAllister

1.

Corresponding author: Andrew S. McAllister, drandrewmcallister@gmail.com

\begin{abstract}
The vascular anatomy of the optic nerve head is complex and sometimes misunderstood. This article reviews the anatomy and relevance to disease processes that affect this vital visual structure.
\end{abstract}

Categories: Neurology, Ophthalmology, Neurosurgery

Keywords: optic disc, anatomy, vasculature, optic nerve, optic neuropathy

\section{Introduction And Background}

The optic head has an intricate and highly vascular blood supply originating from the ophthalmic artery. The anatomy of the vessels in the optic head is important for the understanding of diseases that affect this site and their treatment. Such an example is the obstruction of the optic nerve head arterial blood supply.

\section{Review}

\section{Structure and embryology}

The optic head or disc is a posterior structure, approximately $4 \mathrm{~mm}$ medial from the fovea, is pink-white (paler than retina) and $1.5 \mathrm{~mm}$ in diameter [1-2]. It is the site where optic nerve fibres aggregate to exit the eye as the optic nerve, raised on its edge and depressed centrally where the central retinal vessels enter and leave the eye. As there are no photoreceptors, it is insensitive to light (blind spot). The optic head consists of a surface nerve fibre layer, prelaminar region, lamina cribrosa, and retrolaminar region [3-4]. The vessels at the optic head are exposed to different hydrostatic pressures; anterior to the lamina cribrosa, the tissue is exposed to the intraocular pressure, and posteriorly to the cerebrospinal fluid pressure [2].

The optic head blood supply during development stays relatively the same. The embryonic fissure closure in the second month traps the hyaloid artery centrally, eventually becoming the central retinal artery entering via and supplying the optic head. The choroidal vascular system develops, with the short posterior arteries becoming the principle supply to the optic nerve head [1].

Review began 11/05/2012 Published 02/21/2013

(๑) Copyright 2013 McAllister. This is an open access article distributed under the terms of the Creative Commons Attribution License CC-BY 3.0., which permits unrestricted use, distribution, and reproduction in any medium, provided the original author and source are credited.

\section{Arterial supply}

The optic head is supplied by branches of the ophthalmic artery which originate from the internal carotid artery. Multiple anastomoses between its eleven branches and the external carotid artery allow adequate blood flow to the choroidal and central retinal arteries if the ophthalmic or internal carotid arteries become occluded. Rarely, the central retinal artery comes from the middle meningeal artery [2].

At the optic head, posterior to the laminar cribrosa, the arteries are larger (arterial and arteriolar) than those anteriorly (arteriolar and capillary). At this level, the central retinal artery has the structure of a mediumsized artery and is susceptible to atherosclerosis [1]. This anatomic end artery supplies blood to the inner two-thirds of the retina and surface nerve layer of the optic nerve though provides little to no vascular supply to the prelaminar, lamina and retrolaminar layers [2-6]. 


\section{Cureus}

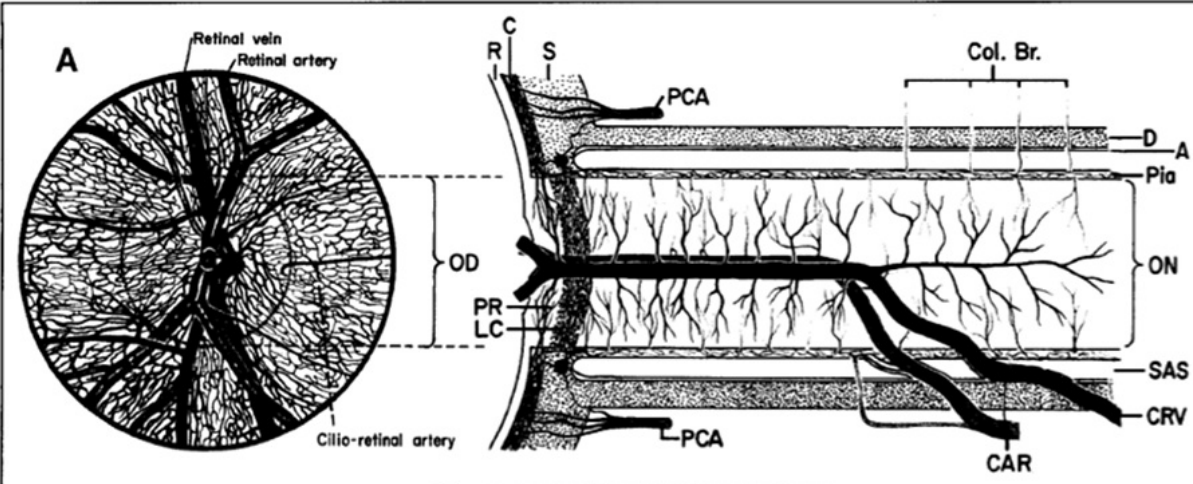

B

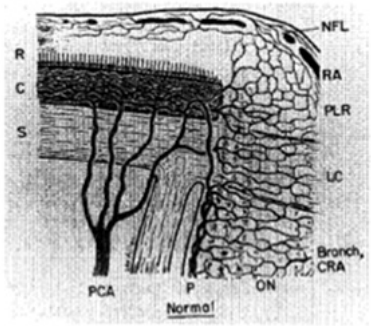

Fig. 1. Schematic representation of blood supply of (a) the optic nerve and (b) optic nerve head. (a, modified from Hayreh SS (1974): Trans Am Acad Ophthalmol Otolaryngol 78:OP240-OP254; b, reproduced from Hayreh, 1978.] A, arachnoid; C, choroid; CAR and CRA, contral retins artery; Col. Br., collateral branches; CRV, central retinal vein; D, duramater; LC, lamina cribrosa; NFL, surface nerve fiber layer of the disc; OD, optic ds: $O N$, optic nerve; P, pia; PCA, posterior ciliary artery; PR and PLR, prelaminar region; R, retina; RA, retinal arteriole; $S$, sclera; SAS, subarachnoid space.

FIGURE 1: Blood supply to optic nerve head

The short posterior ciliary arteries arise from the ophthalmic artery as it crosses the optic nerve, divides into 10 to 20 branches and moves anteriorly around the optic nerve and entwines with the short ciliary nerves [1$2,4]$. The paraoptic vessels enter the sclera medially, laterally and rarely superiorly to the nerve and are the principle blood supply to the optic head [3]. They act as end arteries, and where their supply joins at the optic head, there is a watershed zone susceptible to ischaemia [3]. The high variability in the pattern of blood supply to the optic head means either artery or one alone may supply it. Because of this, occlusive disorders of the same short posterior ciliary artery result in different types of visual loss and manifestations between eyes [3].

To demonstrate the vascular complexity of the optic head, it is generally accepted that the prelaminar region is supplied by the peripapillary choroid composed of the scleral short posterior ciliary system and the recurrent choroidal arteries; the lamina cribrosa is supplied by centripetal branches from the short posterior ciliary arteries either directly or by forming the circle of Zinn and Haller; the retrolaminar region has a peripheral centripetal vascular supply from the pial network of vessels and sometimes an axial centrifugal supply from the central retinal artery [6].

At the prelaminar region, the scleral short posterior ciliary arteries travel through the sclera and the border tissue of Elschnig to reach the prelaminar space without traversing the choroid, supplying traverse capillaries and precapillaries. Recurrent choroidal arteries supply centripetal branches of the peripapillary choroid. Cilioretinal arteries are present in $15-20 \%$ of people from a larger connection between the capillaries of the central retinal artery on the disc surface and posterior ciliary arteries. They are infrequent sources of prelaminar precapillaries and supply the area of retina between the optic disc and macula. It does not anastomose with the central retinal artery or its branches but is important in occlusion of the central retinal artery [1]. Axons on the surface of the optic head receive blood supply from the branches of the central retinal artery, reinforced by the branches from the circle of Zinn [2].

The circles of Zinn and Haller are a rich vascular circle, often incomplete and may be substituted for small branches of the paraoptic short ciliary arteries from the sclera. The circles are contributed by vascular branches of the short posterior ciliary arteries, including the recurrent pial branches from the pial sheath of the leptomeninges, recurrent choroidal arteries, and direct branches to the retrolaminar and laminar parts of the nerve head (these are infrequent). Intraneural branches from the central retinal artery may be a small contribution. In this region, there are also intraseptal vessels of the retrolaminar region which are large precapillaries and capillaries surrounding the axon bundles extending from the lamina cribrosa posteriorly to the intraorbital nerve.

The pial network is made up of recurrent branches from the peripapillary choroid and circle of Zinn and Haler (or the short posterior ciliary arteries in that area) that run posteriorly; and small meningeal branches 
from the central artery as it pierces the pia mater to form an extensive plexus of vessels. The anastomoses between these two vessels are very small, and the central artery of the retina is considered to be an end artery without communication to the choroid [1-3].

Central collateral branches from the central retinal artery as it enters the optic nerve have been described. They may pass anteriorly toward the lamina cribrosa with the central artery, and may pass posteriorly within the optic nerve toward the optic canal and supply the macular nerve fibres [1].

\section{Venous and lymphatic drainage}

The veins draining the optic head and the remaining orbit have no valves, are tortuous and freely anastomose. There are also no lymphatic vessels or lymphoid tissue.

Venous drainage of the optic nerve head is much simpler than its arterial supply. Venules drain into the central vein of the retina formed by tributaries that accompany the arteries, being one-third to one-fourth larger [1]. Occasionally, there are septal veins in the retrolaminar region which drain into pial veins [2]. Some small venules from the prelaminar region or from the nerve fibre layer (opticociliary veins) drain into the choroid [2]. The central retinal vein is susceptible to increased intracranial pressure transmitted by the cerebrospinal fluid as it passes through the subarachnoid space.

The central retinal vein can drain directly into the cavernous sinus or superior ophthalmic vein, but always has a connection with the superior ophthalmic vein [1]. Should the cavernous sinus become thrombosed, the connection with the superior ophthalmic vein allows alternative drainage to the pterygoid venous plexus.

\section{Microscopic structure and blood-tissue barriers}

The veins and arterioles of the optic nerve head have no elastic lamina and few smooth muscle fibres. The endothelial lining the blood vessels is non-fenestrated with tight junctions between them to reduce permeability and form the blood-tissue barrier. However, at the optic head, it is incomplete due to continuity between the extracellular spaces of the choroid and the optic nerve head at the level of the choroid (in the prelaminar region) with a potential route from the choriocapillaris, along Bruch's membrane to the glial sheaths of cells in the prelaminar zone. This pathway is prevented by tight junctions between astrocytes.

\section{Innervation of the vascular supply}

The choroidal arteries supplying the nerve head and the central retinal artery and vein up to the lamina cribrosa have a plentiful supply of vasomotor post-ganglionic sympathetic fibres from the superior cervical sympathetic ganglion [1].

Increased sympathetic activity in the eye is believed to cause arterial vasoconstriction and reduced blood flow, reducing the formation of tissue fluid at the blood-retinal barrier and in formation of aqueous humor. By doing so, the increased production of tissue fluid from the elevation in blood pressure is counteracted [1].

Parasympathetic innervation of the arteries is from the oculomotor nerve via the ciliary ganglion and the facial nerve via the pterygopalatine ganglion. Its vascular role is not fully understood [1].

\section{Clinical features of an obstruction to the optic nerve head arterial blood supply}

The paraoptic short posterior ciliary arteries are the principle blood supply to the optic nerve head and create a watershed zone where flow is determined by resistance within the vessels, intraocular pressure and systemic blood pressure. If the optic nerve head is located within this area and blood flow is reduced, it is vulnerable to ischaemia resulting in anterior ischaemic optic neuropathy (AION) [3]. Common causes are thrombotic/embolic lesions, such as giant cell arteritis (GCA) and other vasculitic diseases, or transient nonperfusion/hypoperfusion, such as in shock, nocturnal hypotension and internal carotid and/or ophthalmic artery stenosis or occlusion [3].

AION presents with pathognomonic symptoms and signs. Patients have sudden and painless visual deterioration, worse when waking in the morning. Their visual acuity may be normal or reduced, and perimetry is the most important functional test. It shows relative or absolute defects over the visual field, the most common is a inferior nasal sectorial defect, next is an inferior altitudinal defect and/or central scotoma, and the least common are optic disc-related field defects [7].

Ophthalmoscopy always identifies optic disc oedema which may be more in one part of the disc than the other $[8,10]$. Often this is associated with splinter haemorrhage(s) at the margin of the disc [11-12]. Asymptomatic optic disc oedema usually precedes visual loss in non-arteritic AION [13]. Oedema resolves by two to three months and is replaced by pallor of the disc, either sectoral or generalised. However, there is little to no relation to the severity of visual loss and degree of disc pallor. Patients with diabetes may have 
telangiectatic-dilated vessels over the disc and increased peripapillary retinal haemorrhages; wrongly treated and diagnosed as proliferative diabetic retinopathy with optic disc neovascularisation [3].

Giant cell arteritis causes a chalky white optic disc oedema in two-thirds of eyes. Once this has resolved, the discs develop cupping of the optic disc indistinguishable from glaucoma except that the neuroretinal rim is pale $[11,14]$.

Fluorescein fundus angiography can be used in the early stages of AION which show filling defects in the optic disc, peripapillary choroid and/or choroidal watershed zones with late disc staining [11, 15-17]. In GCA, the nasal blood supply is often affected [3].

Other features to be aware of are patients with GCA, often middle-aged and older, may have the global symptoms of the disease, although $20 \%$ of cases of AION are due to an occult variety of GCA where there are no systemic symptoms [3]. ESR and CRP may be elevated in arteritic AION, the latter being more sensitive in diagnosis and management of GCA. Visual loss in arteritic AION is massive in $50 \%$ of eyes, with $25 \%$ having $6 / 12$ vision or better [7]. Temporal artery biopsy often diagnoses GCA but is negative in $5 \%$ of cases $[15,18]$. In non-arteritic AION, a history of amaurosis fugax is highly suggestive of embolic phenomenon preceding permanent blindness $[3,19]$. The aggressive use of arterial hypotensives combined with nocturnal hypotension can also cause progressive visual field deterioration [20-21].

Central retinal artery occlusion also causes sudden unilateral loss of vision, but the optic head is normal in $17 \%$ of cases within one week of occlusion [22]. Often, the disc is pale (39\%) and some may have initial hyperaemia. Rarely is there a whitish accumulation of retrograde axoplasmic flow. Over time, $63 \%$ of eyes develop pale discs by one month, $79 \%$ after two months and $91 \%$ by four months [10].

\section{Conclusions}

The optic head receives its intricate and variable end arterial blood supply from the posterior choroidal circulation. Understanding its vascular anatomy is important in clinical practice, for understanding pathology, disease processes, presentation as well as their treatment, and why it is highly susceptible to ischaemia.

\section{Additional Information \\ Disclosures}

Conflicts of interest: In compliance with the ICMJE uniform disclosure form, all authors declare the following: Payment/services info: All authors have declared that no financial support was received from any organization for the submitted work. Financial relationships: All authors have declared that they have no financial relationships at present or within the previous three years with any organizations that might have an interest in the submitted work. Other relationships: All authors have declared that there are no other relationships or activities that could appear to have influenced the submitted work.

\section{References}

1. Snell R, Lemp M: Clinical anatomy of the eye. 2nd ed . Blackwell Science, Inc, 1998

2. Bron A, Tripathi C, Tripathi B: Wolff's anatomy of the eye and orbit. 8th ed . Chapman and Hall, New York; 1997.

3. Hayreh SS: Anterior ischemic optic neuropathy. Clin Neurosci. 1997, 4:251-63.

4. Mackenzie PJ, Cioffi GA: Vascular anatomy of the optic nerve head . Can J Ophthalmol. 2008, 43:308-12. 10.3129/i08-042

5. Hayreh SS: Blood supply of the optic nerve head and its role in optic atrophy, glaucoma and oedema of the optic disc. Br J Ophthalmol. 1969, 53:721-48.

6. Hayreh SS: The blood supply of the optic nerve head and the evaluation of $\mathrm{t}$ - myth and reality . Prog Retin Eye Res. 2001, 20:563-93. 10.1016/S1350-9462(01)00004-0

7. Hayreh SS, Podhajsky PA: Visual field defects in anterior ischaemia optic neuropathy . Doc Ophthalmol Proc Series. 1979, 19:53-71. 10.1007/978-94-009-9611-3_6

8. Hayreh SS, Zimmerman MB: Management of giant cell arteritis: Our 27-year clinical study. New light on old controversies. Ophthalmologica. 2003, 217:239-59. 10.1159/000070631

9. Hayreh SS: Anterior ischaemic optic neuropathy: Differentiation of arteritic from non-arteritic type and its management. Eye. 1990, 4:25-41.

10. Hayreh SS, Podhajsky PA, Zimmerman MB: Ocular manifestations of giant cell arteritis. Am J Ophthalmol. 1998, 125:509-20. 10.1016/S0002-9394(99)80192-5

11. Hayreh SS: Anterior ischaemic optic neuropathy II. Fundus on ophthalmoscopy and fluorescein angiography Br J Ophthalmol. 1974, 58:955-63.

12. Hayreh SS: Anterior ischaemic optic neuropathy: Differentiation of arteritic from non-arteritic type and its management. Eye. 1990, 4:25-41.

13. Hayreh SS: Anterior ischemic optic neuropathy V. Optic disc edema an early sign . Arch Ophthalmol. 1981, 99:1030-40.

14. Hayreh SS: Segmental nature of choroidal vasculature. Br J Ophthalmol. 1975, 59:631-48. 10.1136/bjo.59.11.631 


\section{Cureus}

15. Hayreh SS: In vivo choroidal circulation and its watershed zones. Eye. 1990, 4:283-89. 10.1038/eye.1990.39

16. Hayreh SS: Anterior ischemic optic neuropathy. Arch Neurol. 1981, 38:675-78.

17. Hayreh SS: Inter-individual variation in blood supply of the optic nerve head. Its importance in various ischemic disorders of the optic nerve head, and glaucoma, low-tension glaucoma and allied disorders. Doc Ophthalmol. 1985, 59:217-46. 10.1007/BF00159262

18. Hayreh SS: Physiological anatomy of the choroidal vascular bed . Int Ophthalmol. 1983, 6:85-93. 10.1007/BF00127636

19. Portnoy SL, Beer PM, Packer AJ, Van Dyk HJ: Embolic anterior ischemic optic neuropathy . J Clin Neuroophthalmol. 1989, 9:21-5.

20. Hayreh SS, Podhajsky PA, Zimmerman MB: Nocturnal arterial hypotension and its role in optic nerve head and ocular ischemic disorders. Am J Ophthalmol. 1997, 117:603-24.

21. Hayreh SS: Systemic aterial blood pressure and the eye . Eye. 1996, 10:1-24. 10.1038/eye.1996.3

22. Hayreh SS, Zimmerman MB: Fundus changes in central retinal artery occlusion . Retina. 2007, 27:276-89. 